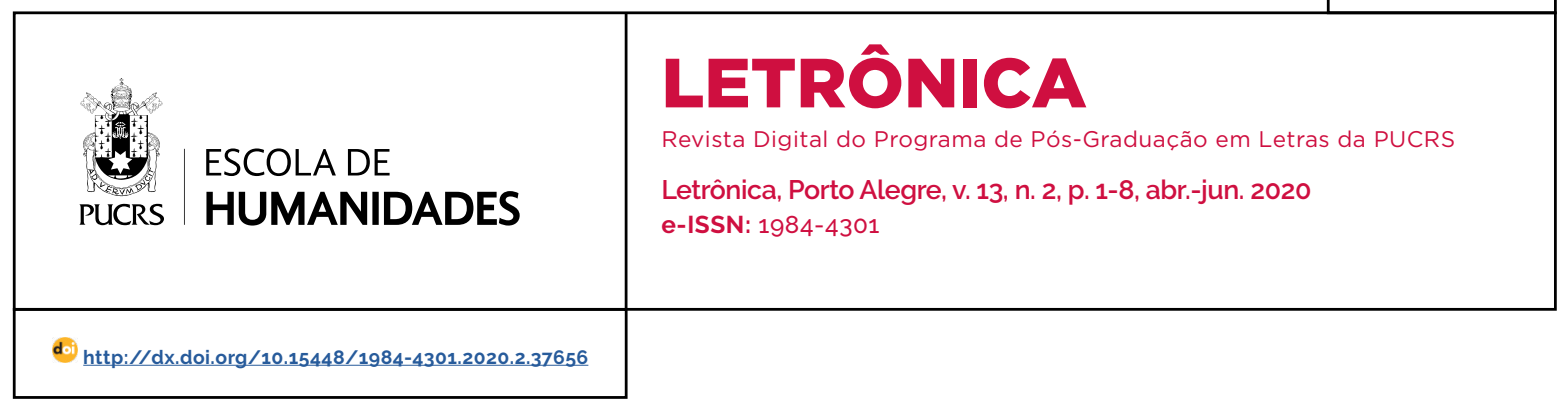

\title{
Discursos (In)tolerantes y Democracia Pluralista
}

\author{
(In)Tolerant Discourses and Pluralist Democracy \\ Discursos (In)tolerantes e Democracia Pluralista
}

Juan Manuel López-

Muñoz ${ }^{1}$

orcid.org/0000-0002-2208-2540

imanuel.lopez@uca.es

\section{Maria da Glória Corrêa \\ di Fanti ${ }^{2}$ \\ orcid.org/0000-0002-5399-5377 \\ gloria.difanti@pucrs.br}

\section{Bárbara Luzia Covatti \\ Malcorra ${ }^{2}$}

orcid.org/0000-0001-5710-7130

barbara.malcorra@edu.pucrs.br

Recebido em: 12 mar. 2020 Aprovado em: 13 abr. 2020 Publicado em: 13 ago. 2020.

\section{(c) (1)}

Artigo está licenciado sob forma de uma licença Creative Commons Atribuição 4.0 Internacional.
Parece que existe un consenso general respecto a la idea de que el desarrollo de las nuevas tecnologias de la comunicación y, concretamente, el desarrollo de las plataformas digitales de socialización, han reforzado las tensiones entre grupos, asi como la preocupación de las sociedades actuales por la cuestión de la seguridad ante la propagación de discursos y prácticas intolerantes (SICARD, 1998, p. 9). Las instituciones, públicas y privadas, están cada vez más sensibilizadas contra la intolerancia, por temor a que ésta pueda llegar a desestabilizar el orden social vigente y generar desconfianza respecto al consumo de las nuevas tecnologias entre los usuarios. De este entendimiento parte, sin duda, la motivación del presente número de la revista Letrônica, así como de la ingente literatura científica y periodística en los últimos años sobre el tema.

Además, numerosos observatorios de los discursos intolerantes en la red han sido creados, como el de la Asociación Start Turning Off Prejudice Heal Attitudes Through Education (STOP HATE Crime and discrimination: www.stophate.com/), el del Proyecto Centinela para la Prevención del Genocidio (hatebase.org/), el del Observatorio Español del Racismo y la Xenofobia (OBERAXE www.mitramiss.gob.es/oberaxe/es/index.htm), o el Observatorio Proxi contra la Xenofobia y la Intolerancia en medios digitales (www.observatorioproxi.org/), por poner algunos ejemplos destacables.

Por otra parte, la Asamblea Parlamentaria del Consejo de Europa y otras instituciones supranacionales como la Organización de los Estados Americanos, el Parlamento Europeo, la Comisión Europea contra el Racismo y la Intolerancia (ECRI), la Asamblea General de las Naciones Unidas y La Organización de las Naciones Unidas para la Educación, la Ciencia y la Cultura destinan, desde hace muchos años, esfuerzos y fondos para la publicación de tratados internacionales como el Convenio sobre la Ciberdelincuencia (2001), el Manual On Hate Speech (2009), la Convención Interamericana contra toda forma de discriminación e intolerancia (2013) o, en fin, más reciente y concretamente, el manual Cómo contrarrestar el discurso de odio en línea (2015).

\footnotetext{
Universidad de Cádiz (UCA), Cádiz, Espanha.

Pontificia Universidade Católica do Rio Grande do Sul (PUCRS), Porto Alegre, RS, Brasil.
} 
La prensa, la radio y la televisión tampoco son ajenos a esta responsabilidad compartida con los investigadores y las instituciones (inter) nacionales ante la necesidad de destacar las fortalezas de la vida social digital y de permitir que se expresen los miedos, evitando las medias verdades tanto como los catastrofismos o la amplificación desmesurada del fenómeno de la intolerancia (SICARD, 1998, p. 149-168).

Sin quitar mérito, obviamente, a todos los esfuerzos de los investigadores que nos precedieron, nosotros creemos que la ventaja del presente volumen está en que hemos privilegiado la observación y el estudio frente a la condena, contribuyendo a una mayor visibilidad del sujeto anatematizado respecto al sujeto intolerante, y una mayor legitimidad (mayor difusión) de los discursos tolerantes respecto a los intolerantes. El estudio anecdótico de discursos intolerantes cede aqui el paso a estudios de la (in)tolerancia con una perspectiva más amplia que, además, permite dar a conocer en profundidad las claves discursivas, culturales y socio-económicas del fenómeno, destacando a ciertos grupos considerados históricamente como víctimas de la intolerancia.

Claro que también tienen cabida aqui análisis de casos concretos de discursos intolerantes que encontramos en el actual clima de crispación social, pero teniendo en cuenta que ninguno de ellos debe entenderse como gesto intolerante aislado (IMBLEAU, 2003, p. 311).

En efecto, entendemos que un debate sobre unas caricaturas más o menos blasfemas o sobre un discurso extremista, populista, a cargo de un político o de una persona influyente concreta que en un determinado lugar extiende difamaciones, injurias o propaganda discriminatoria, siendo necesario, no debe distraer la atención de los investigadores, por exceso de focalización microscópica, ni servir al impulso de estrategias de limitación de la libertad de expresión. ${ }^{3}$

Sobre todo, los análisis que reunimos en este volumen, incluso aquellos que están anclados en un discurso concreto más o menos claramente criminal, no nos alejan del objetivo primordial, que es, según creemos, dar las claves que permitan a las sociedades liberarse de los estereotipos y patrones de comportamiento (también lingüisticos) ligados a las relaciones de poder institucionalizadas ${ }^{4}$ y que están en la base de la intolerancia y el odio.

¿La crisis de tolerancia actual está generada por las TIC? ¿Podemos hablar de "riesgo tecnológico mayor" (LAGADEC, 1981), marcando una ruptura entre la intolerancia de ayer y la de hoy? ¿Acaso la gravedad del problema actualmente no tiene nada en común con el fenómeno de los discursos intolerantes anteriores al desarrollo de las redes sociales? ¿Cuáles son las claves socio-económicas, culturales, políticas de la intolerancia hoy, dentro y fuera del ciberespacio? Tales son las preguntas a las que los autores reunidos en este volumen intentan responder directa o indirectamente.

Está claro que la intolerancia no es un fenómeno nuevo, al estar ligada a órdenes sociales basados tradicionalmente en el antagonismo (la relación entre enemigos). Mouffe (2010)5 explica que todo orden social antagonista tiene un origen politico hegemónico, basado en prácticas coyunturales que, sin embargo, en un momento dado, en un lugar determinado, se establecieron como permanentes y fueron tratadas, a continuación, como procedentes de un orden natural.

La intolerancia puede explicarse entonces como consecuencia de la aceptación de un orden social como orden natural. Ahora bien, insiste Mouffe (op. cit, p. 20), el orden social siempre podría haber sido y aún puede ser diferente, en tanto que se estableció desde su origen a partir de la exclusión de otras posibilidades. En democracia, el orden social nunca es la manifestación de un orden natural, sino el resultado de un acuerdo humano, circunstancial y, por tanto, se trata de un orden revisable, discutible.

El orden social basado en antagonismos siempre

\footnotetext{
3 CARRILLO DONAIRE, Juan Antonio. La protección de los derechos frente a los discursos del odio: del Derecho represivo a las politicas públicas antidiscriminatorias. In: ALONSO, L.; VÁZQUEZ, V. (dir.), 2017. p. 15-38.

4 Ver, entre otros, Butler (2009, p. 47-97).

5 MOUFFE, Chantal. Politique et agonisme, Rue Descartes, v. 67, n. 1, 2010, p. 18-24. Aunque el artículo no es reciente (publicado en 2010), estimamos que puede tener mucha relevancia en el contexto del presente número y en la situación global actual.
} 
puede ser desarticulado y transformado en otro orden diferente, sostenido por una dinámica agonista, según defiende Mouffe (ibid.). Lo que está en juego en la dinámica agonista, aclara esta filósofa y politóloga belga, es la configuración misma de las relaciones de poder que estructuran el orden social hegemónico. En una confrontación agonista, los proyectos opuestos nunca pueden ser reconciliados racionalmente, sino simplemente aceptados en un régimen que promueva la tolerancia y la cohabitación de estos.

La relación agonista ${ }^{6}$, además de promover la tolerancia, tienen la ventaja de que no necesita destruir el orden existente ni tampoco partir de cero, sino simplemente recuperar aquellos principios de igualdad y libertad proclamados que todavía no (o ya no) están siendo aplicados efectivamente por las democracias liberales modernas.

Aplicado a las interacciones cotidianas, el pluralismo agonista significa, entendemos nosotros, que aquella persona que no comparte nuestra identidad no será considerada como un enemigo al que derrotar sino como un oponente cuya existencia es legitima. El individuo intolerante podrá defender vigorosamente sus ideas y sus discursos de su grupo dominante, pero nunca impedirá al individuo de un grupo dominado ejercer su derecho a defender las ideas propias y nunca cuestionará la legitimidad del interlocutor.

Mouffe (op. cit., p. 19) precisa, respecto a este razonamiento, que es imposible que la categoria del enemigo desaparezca como tal, sino que está constituida por un Ellos (los sujetos intolerantes, quienes no respetan los principios democráticos básicos de igualdad y libertad) frente a un Nosotros (formado por grupos que, aunque opuestos a Ellos, no cuestionan los principios de la democracia pluralista).

Partiendo de tal distinción entre antagonismo (relación entre enemigos) y agonismo (relación entre opuestos no enemigos), Mouffe (ibid.) defiende la idea de que la confrontación agonista, lejos de representar un peligro para la democracia es, en realidad, la condición óptima para la existencia de una democracia auténtica, radicaly, por tanto, según creemos, la condición óptima para poner fin a las prácticas intolerantes. La democracia radical implica perseguir ciertas formas de consenso, pero garantizando que pueda ocurrir una cierta discordia o conflicto entre Ellos y Nosotros, de tal manera que los ciudadanos tengan la oportunidad de expresarse libremente en un clima de tolerancia.

La idea de una identidad que sería la única apropiada para un grupo y que habitualmente sirve como punto de origen de los discursos intolerantes, debe ser reemplazada por la idea de la aceptación de una multiplicidad de identidades que cohabitan en un espacio agonista. Lo importante, para Mouffe (op. cit., p. 22), es garantizar la expresión de la pluralidad y evitar cualquier proceso intolerante de castigo, interrogatorio, culpabilización o intimidación del oponente.

Mouffe aboga por una radicalización de la democracia mediante el desarrollo de un nuevo espiritu democrático entre los ciudadanos basado en el compromiso permanente con una dinámica agonista que haga que todos los intentos de cerrar los debates y aplastar al oponente sean vanos, cuando no imposibles (op. cit., p. 23).

Más o menos en esta misma línea, pero apuntando más lejos que a la importancia de la pluralidad, a una revisión de la noción misma de identidad, cabe destacar el librito de François Jullien, titulado Il n'y a pas d'identité culturelle (Paris: l'Herne, 2016), traducido recientemente al castellano por Imanol Zubero (La identidad cultural no existe, Taurus, Madrid 2017). Tal revisión permitiría acabar con el debate de la intolerancia, si consideramos, siguiendo a Jullien, que ésta parte de la premisa falaz de la existencia de identidades culturales. Según el citado filósofo, la existencia de identidades colectivas diferentes entre si, pretendidamente uniformes, las cuales se encontrarian en situaciones de riesgo de desaparición o disolución en el sistema de

6 En el artículo citado, Mouffe desarrolla el modelo de "pluralismo agonista" (que ya abordó en algunos de sus libros anteriores: The Democratic Paradox, Londres: Verso, 2000 y On the Political, Abingdon - Nueva York: Routledge, 2005). Este modelo se basa en la premisa de la distinción entre las categorias de enemigo y oponente.

7 Véase también François Jullien (2012). 
globalización actual, es una simple creencia; no es un hecho. Abandonar tal premisa falsa requiere entonces dejar de lado las nociones de identidad $y$ de diferencia, sustituyendo, según propone el autor, esta última por la de "intervalo" (écart ${ }^{8}$ ) y aquella por la de "recursos" (ressources).

De hecho, este autor considera dos posibles concepciones de identidad: por un lado, la singular, subjetiva, que es válida en contextos conversacionales concretos (entendemos: "yo", diferente de "tú") y, por otro lado, la colectiva, más o menos objetiva, de naturaleza cultural, que en realidad no existe $\mathrm{o}$, al menos, no existe como un "valor" propiamente dicho, articulador de un grupo, sino como un mero marco mitológico. La ventaja del concepto jullieniano de "recursos" en lugar de "identidad" estriba en que permite ir más allá del multiculturalismo, al plantear que las culturas son bienes al alcance de cualquier individuo, independientemente del origen de este. Los "recursos" (las supuestas identidades) no son valores, que requeririan aprobación o conversión, sino medios disponibles para resolver una necesidad o llevar a cabo una determinada acción.

El autor desarrolla a lo largo de su librito las nociones de "intervalo" (écart) y de "recurso" (ressource), las cuales impiden clasificar o separar a los grupos atendiendo a rasgos socioculturales distintivos, invitando, al contrario, a pensarlos en un modo interactivo y dinámico, sujetos a constantes mutaciones y transformaciones. Dicho de otro modo, las culturas son recursos, riquezas disponibles para todos, y no son valores o marcos cognitivos de referencia. Son recursos que se aprovechan, se intercambian, pero no se venden ni requieren carné de afiliado. En este orden de ideas "defender" unos determinados recursos (unas determinadas verdades frente a otras), no significa tanto protegerlos como explotarlos, ponerlos al servicio de todos los seres humanos.

Esta visión dinámica de la necesidad de la interrelación entre grupos basada en lo compartido contrasta con la vieja visión predominante hasta ahora que pone su foco en las diferencias y las semejanzas entre grupos. El "intervalo" de Jullien no opone dos elementos, promoviendo a uno frente al otro de forma antagónica, sino que precisa de ambos lados. El "intervalo" crea un espacio exploratorio común que permite el roce y la comparación, pero no la exclusión. Por decirlo de un modo simple, no se trata de distinguir, por poner un ejemplo, hombres de mujeres, ricos de pobres, blancos de negros, colonizadores de indios, sino de poner de relieve el espacio de su relación, es decir, de poner de relieve ese intervalo que tienen en común dos partes de una misma cosa en el que cada uno puede empezar a entender al otro.

Tal cambio conceptual lleva al autor, François Jullien, a redefinir la idea de diálogo entre culturas ${ }^{9}$, que debería entenderse como recorrido y no como intento de asimilación y, en fin, que debería basarse en ejercicios de análisis y no de sintesis.

Por último, volviendo a esta idea del carácter mitológico de la identidad al que aludiamos un poco más arriba, podemos convenir con Moulay y Rebischung (2011, p. 3-5) que la intolerancia al pretendido enemigo es una reacción emocional ante las diferencias de este y que el miedo y el odio resultantes son emociones que no están asociadas a una experiencia vivida, sino a un rumor o a una creencia más o menos extendida. O sea, la intolerancia puede ser una respuesta física, corporal, más o menos incontrolable, pero tiene su origen principalmente en una cuestión mental, discursiva y cultural, ideológica y, sobre todo, mitológica (CARRERA, 2019, p. 17), siendo, por consiguiente, perfectamente controlable. Controlable, decimos, no suprimible, "pues quienes tratan de suprimir llal mito-diversidad, exigiendo a todas las personas vivir bajo el mismo mito, que unos comparten y otros no, [se] deshumanizan, [dando lugar al los llamados totalitarismos" (ibidem).

\footnotetext{
8 El autor emplea en francés el término de "écart" derivado del verbo écarter, que normalmente designa la acción por la cual dos partes de una misma cosa se desvían más o menos una de la otra (las negritas son nuestras). Entendemos, siguiendo a Jullien, que écart implica un entre dos, es decir, una dinámica de relación entre dos puntos o limites dados. El traductor para la edición en español ( $L a$ identidad cultural no existe. Taurus, Madrid 2017), Imanol Zubero Beaskoetxea, propone como equivalente el término "brecha", que nosotros descartamos precisamente por sus valores negativos (ligados a ideas de herida, de rotura en un frente de combate o de resquicio por donde algo empieza a perder su seguridad).

9 Véase François Jullien (2008).
} 
En la comunicación mediada por ordenador o dispositivo electrónico, el problema de los antagonismos basados en (falsas) identidades culturales se vuelve más complejo por la intercalación de la cibercultura frente a la cultura offine, cada una con su correspondiente memoria de los usos escritos y orales (LOPEZ-MUÑOZ, 2019).

Esta cibercultura es una masa de prácticas y conocimientos compartidos (un tecno-imaginario, BALANDIER, 1985), que trasciende lo lingüistico y lo cultural (en sentido tradicional, ligado a una lengua materna o adquirida), fabricándose en el día a día de las interacciones en la red. Por la red interactuamos de un modo diferente (ASSOULINE, 2012), con una postura corporal diferente (SZUNDY et al., 2016, p. 356), pasando de una conversación a otra, de una búsqueda a otra, unas veces con un objetivo concreto y otras veces no.

Lo de la postura corporal diferente no es un aspecto menor, como podría parecer, pues las emociones tienen una importante dimensión corporal, además de afectiva y cognitiva. Disponemos de un variado código corporal/ gestual para manifestar las emociones más básicas, como el odio y el rechazo, pero ¿cómo se expresan estas en una situación donde el cuerpo queda prácticamente abolido o ausente ${ }^{10}$, y que el contacto entre interactuantes de la conversación se reduce a toques en el teclado de una máquina, con nuevas relaciones de poder al mismo tiempo que los espacios de contacto se amplian, llegando a suceder conversaciones en espacios (dormitorios ${ }^{11}$, cuartos de baño, despachos) normalmente reservados para la intimidad, la soledad o el quehacer profesional. ${ }^{12}$

Respecto a las nuevas relaciones de poder que se establecen en el ciberespacio, y que son fuente de discursos intolerantes, Catherine Blaya considera que actualmente los "tecno-poderosos" (BLAYA, 2019) son aquellos individuos jóvenes (principalmente adolescentes) que poseen los conocimientos tecnológicos e informáticos y las habilidades digitales (tales como prácticas de publicación, difusión, referencia e intercambio), más allá de los condicionamientos sociológicos tradicionales en el mundo offline (la clase social, el género, o el origen étnico). El problema de los discursos intolerantes en la red es singular por el motivo de que, al contrario que en las situaciones offline donde estos discursos son generalmente tan violentos como fugaces, en la red en cambio son durables, trazables, y están caracterizados por una gran interactividad (viralizacion, likes). ${ }^{13}$

A modo de ilustración de las reflexiones precedentes, el presente volumen contiene dieciocho artículos. En Tonos intolerantes en discursos de grandes medios de comunicación brasileños: un estudio dialógico, Maria da Glória Corrêa di Fanti y Juan Manuel López-Muñoz analizan discursos intolerantes publicados por la revista Isto É y el periódico Folha de S. Paulo contra la presidenta Dilma Rousseff, durante el proceso de impeachment. Tras este artículo, Luiz Fernando Boaventura y Ernani Cesar de Freitas, en Encenação e ubiquidade no Twitter: a intolerância dos discursos sobre Marielle Franco, investigan una conversación en Twitter sobre el asesinato de Marielle Franco y Anderson Gomes el 14 de marzo de 2018.

En el artículo Minions nas telas e bolsominions na vida: uma análise bakhtiniana, Luciane de Paula y Natasha Ribeiro de Oliveira reflexionan sobre el término "bolsominion" en Facebook, observando la articulación entre discurso, vida y arte. Tamiris Machado Gonçalves, en Charge

\footnotetext{
10 "Lo digital a menudo se asocia con prácticas 'sin rostro', pero el anonimato de los usuarios no significa que el abusador y la víctima no se conozcan", precisa C. Blaya (2012) en línea "Cyberharcèlement et climat scolaire: les premières données de l'enquête" de C. Blaya", Ihttp://www.touteduc.fr/fr/archives/id-5848-cyberharcelement-et-climat-scolaire-les-premieres-donnees-de-l-enquete-de-c-blayal. 11 Véase el concepto de Bedroom culture en Mari-Anne Paveau (2016, p. 13). [https://hal.archives-ouvertes.fr/hal-01423473/document]. 12 Cécile Dolbeau-Bandin habla en estos casos de efecto cockpit, basándose en la analogia con los pilotos de combate que, en una cabina protegida y muy lejos de su objetivo, los cuales no se dan cuenta directamente del daño causado. La autora cita, a este respecto, el «Rapport Cyberharcèlement: Risque du virtuel, impact dans le réel» del Observatoire des Droits de l'Internet, 2009, URL: http://economie.fgov.be/fr/binaries/Boekcyberpestenfr_tcm326-271185.pdf, p. 17 [https://www.mesdatasetmoi-observatoire.fr/article/parlons-enfin-de-cyberharcelement\#anchor13]

13 Los discursos intolerantes en la red se caracterizan por la velocidad y la viralización con las que se pueden difundir, con el simple gesto de un solo clic. Los discursos intolerantes pueden transmitirse, "gustar" y compartirse incluso miles de veces. Y peor aún, son perdurables, pudiendo llegar a perseguir a su víctima mucho después del hecho. Véase, entre otros, Cécile Dolbeau-Bandin https://wwww. mesdatasetmoi-observatoire.fr/article/parlons-enfin-de-cyberharcelement\#anchor13
} 
com contorno intolerante: uma análise discursiva, analiza una caricatura de Roque Sponholz, publicada en 2011, en los medios digitales brasileños. Evandra Grigoletto y Thiago Alves França, en el artículo Discursos de resistência à intolerância pela censura: o caso da propaganda do Banco do Brasil, analizan la intolerancia a través de la controversia generada por una pieza publicitaria cuya exhibición oficial fue impedida por el presidente Jair Bolsonaro.

En Fake news e pós-verdade na construção do Neoconservadorismo no Brasil pós-2013 e os efeitos nas eleições de 2018, Mauri de Castro Azevedo y Marcus Antônio Assis Lima discuten sobre el aumento de ideas extremistas en la sociedad brasileña por efecto de la difusión masiva de noticias falsas y de discursos politicos intolerantes en las redes sociales en las últimas elecciones presidenciales. También se ocupan de las redes sociales Marcelo Vinicius Costa Amorim y Antônio Fernandes Júnior quienes, en (Diz) positivo para matar: uma análise sobre o discurso de ódio no Brasil contemporâneo, estudian la arqueo-genealogía de los discursos de odio y los efectos de estos en los procesos de subjetivación.

Contemplando cuestiones de género, por un lado, Bruna Vitória Tejada y Luciana lost Vinhas, en Aquilo que não pode deixar de ser dito: o efeito de pré-construido do discurso machista, investigan la noción de preconstruido desde la perspectiva del Análisis del Discurso de tradición francesa. Por otro lado, Fábio de Castro Dias, Marina Alvarenga Botelho y Marco Antonio Villarta-Neder, en el artículo $A$ violência da palavra em ausência no processo discursivo de representação das mulheres: uma análise do Projeto de Lei 01-00352/2019. analizan la representación de la mujer en Proyecto de ley del Ayuntamiento de São Paulo. Por su parte, cerrando este apartado, Martha Júlia Martins de Souza, en Feminismo e intolerância: uma análise das leis estaduais do estado de Roraima, investiga cuestiones de género en el entorno escolar a partir de las leyes 1245/2018 y 1323/2019.

En el artículo Documentos jurídicos, "cura gay" e legitimação do heterossexismo: uma análise discursiva do PDC 234/2011, Gabriel
Merlim Moraes Villela, Maria Cristina Giorgi, Fabio Sampaio de Almeida y Dayala Paiva de Medeiros Vargens investigan la función que desempeñan documentos jurídicos al legitimar ciertas prácticas heterosexistas que alimentan discursos de odio e intolerancia. Welton Pereira e Silva, en Discursos criminalizáveis: proposta de conceituação a partir de cartas de ameaça, se interesa por el imaginario socio-discursivo en el que se basan los argumentos intolerantes presentes en dos cartas de amenaza con motivación homofóbica. En el artículo Discursos politico-religiosos como armas de guerra: heteroterrorismo em ação contra sexualidades dissidentes, Alexandre de Oliveira Fernandes y Luciano Fernandes de Souza sostienen que la interpelación, en discursos político-religiosos extremistas, inscribe a los disidentes sexuales en un escenario de heteroterrorismo.

La cuestión de la libertad y la autonomía de las escuelas, de los estudiantes y los docentes frente al Proyecto de Ley Escuela Sin Partido (PL 7.180/2014) está en el origen del artículo firmado por Rosely Diniz da Silva Machado y Letícia Rosaura da Silva Maass con el título de We don't need no education: escola sem partido de quem e para quem? Por su parte, Ilmara Coutinho, en Reflexões sobre leitura e ensino em tempos de pós-verdade, reflexiona sobre el desafio de la formación lectora en los tiempos actuales, defendiendo la enseñanza de la lectura como acto político, al permitir pensamientos críticos respecto a las contradicciones sociales contemporáneas.

En Temperança de Tonus: agora vai ser assim contra a intolerância, Daniel de Oliveira Gomes establece una relación entre la poesía de Leonardo Tonus con la figura de la Templanza del Tarot, inspirándose en los trabajos de Milton Santos y Zigmunt Bauman. Elisa Marchioro Stumpf, en Eufemismo: um fenômeno multifacetado no cruzamento entre lingua e cultura, trata temas como la (des)cortesía y la violencia verbal. Finalmente, Ana Raquel Motta, en el artículo "Comunicação não-violenta" pelas lentes da Linguística: embates no combate à intolerância, destaca la contribución de los estudios lingüísticos al desarrollo de la 
noción de comunicación no violenta.

Además de los artículos mencionados, el presente número incluye dos reseñas. Por un lado, Luiz Augusto Ely presenta una recensión de la obra Análisis del discurso, disciplina interpretativa en interdisciplinariedad: violencia y estudios ético-políticos de los discursos, organizado por Graciana Vázquez Villanueva y Pablo Stecher y publicado por la Facultad de Filosofía y Letras de la Universidad de Buenos Aires en 2019. Por último, Baal Delupi reseña el libro colectivo de Politica prática, organizado por Everton Maciel, Sergio Corrêa y Tiaraju Andreazza y publicado por la Universidad Federal de Amapá en 2020.

El conjunto de los trabajos aquí presentados estimula el debate y aboga por la reconstrucción del orden social, según lo postulado por Mouffe (2010), con el objetivo de contribuir a la implementación de una democracia plural, resistente frente a las prácticas discursivas intolerantes. En resumen, con este número de Letrônica defendemos la importancia del respeto a la diversidad de posicionamientos y de modos de vivir, sin impedir la discusión ni silenciar las contradicciones.

\section{Referencias}

ASSOULINE, Pierre. La métamorphose du lecteur. Le Débat, n. 170, p. 78-89, 2012. https://doi.org/10.3917/ deba.170.0078

BALANDIER, Georges. Le Détour: Pouvoir et modernité. Paris: Fayard, 1985.

BLAYA, Catherine. Les jeunes et la violence sur Internet. Paris: Éditions Nouveau Monde, 2019.

BLAYA, Catherine. Cyberharcèlement et climat scolaire: les premières données de l'enquête. Disponivel em: http://www.touteduc.fr/fr/archives/ id-5848-cyberharcelement-et-climat-scolaire-les-premieres-donnees-de-l-enquete-de-c-blaya. ToutEduc, 2012.

BUTLER, Judith. Lenguaje, poder e identidad. Madrid: Sintesis, 2009.

CARRERA, Oscar. Mitología humana. Madrid: Ápeiron Ediciones, 2019

CARRILLO DONAIRE, Juan Antonio. La protección de los derechos frente a los discursos del odio: del derecho represivo a las políticas públicas antidiscriminatorias. In: ALONSO, L.; VÁZQUEZ, V. (dir.). Sobre la libertad de expresión y el discurso del odio. Sevilla: Athenaica, 2017. p. 15-38.
CONSEIL DE L'EUROPE. 23.XI.2001. Convênio sobre la ciberdelincuencia. Disponivel em: https://www.oas. org/juridico/english/cyb_pry_convenio.pdf. Acesso em 03 jan. 2020.

CONVENCIÓN INTERAMERICANA CONTRA TODA FORMA DE DISCRIMINACIÓN E INTOLERANCIA. 2013. Disponivel em: http://www.oas.org/es/sla/ ddi/docs/tratados_multilaterales_interamericanos_A-69_discriminacion_intolerancia.pdf. Acesso em: 03 jan. 2020

DOLBEAU-BANDIN, Cécile. Parlons (enfin) de cyberharcèlement ! L'Observatoire, mesdatasetmoi.fr, 05/10/2017. Disponivel em: https://www.mesdatasetmoi-observatoire.fr/article/parlons-enfin-de-cyberharcelement\#anchor13. Acesso em: 03 jan. 2020.

IMBLEAU, Martin. La négation du génocide nazi, liberté d'expression ou crime nazi? Le négationnisme de la Shoah en droit international et comparé. Paris: Budapest, Turin, l'Harmattan, 2003.

JULLIEN, François. Il n'y a pas d'identité culturelle. Paris: l'Herne, 2016.

JULLIEN, François. L'écart et l'entre. Paris: Galilée, 2012.

JULLIEN, François. De l'universel, de l'uniforme, du commun et du dialogue entre les cultures. Paris: Fayard, 2008.

LAGADEC, Patrick. Risque technologique majeur Politique et processus de développement. Paris: Pergamon, 1981.

LÓPEZ-MUÑOZ, Juan Manuel. Identidad y lengua en el ciberespacio: ¿existe una conciencia lingüistica digital? Gragoatá, v. 24, n. 48, p. 117-135, 2019. https:// doi.org/10.22409/gragoata.v24i48.33622

MANUAL CÓMO CONTRARRESTAR EL DISCURSO EL DISCURSO DE ODIO EN LÍNEA. 2015. Disponível em: https://unesdoc.unesco.org/ark:/48223/ pfooo0233231. Acesso em: 03 jan. 2020.

MOUFFE, Chantal. Politique et agonisme. Rue Descartes, v. 67, n. 1, 2010, p. 18-24. https://doi. org/10.3917/rdes.067.0018

MOUFFE, Chantal. On the political, Abingdon, Nueva York: Routledge, 2005.

MOUFFE, Chantal. The democratic paradox. Londres: Verso, 2000.

MOULAY, Maurice; REBISCHUNG, Thomas. Comprendre l'émotion, Paris: MaxMilo, 2011.

PAVEAU, Mari-Anne. Éthique du discours numérique. Linguas e Instrumentos Linguisticos (Brésil), RG Editora, 2016, 37, p.177-210. Disponivel em: https://hal. archives-ouvertes.fr/hal-01423473/document.

SICARD, Marie-Noële. Entre médias et crises technologiques: les enjeux communicationnels, Paris: Presses Universitaires du Septentrion, 1998.

SZUNDY, Paula; CARRĖRA, Tatianne; NASCIMENTO, Luciana Marino. Leitores-navegantes de textos e hipertextos da literatura. Gragoatá, n. 40, v. 1, p. 354-379, 2016. 
WEBER, Anne. September 2009. Manual on hate speech. Disponivel em: http://icm.sk/subory/Manual_on_hate_speech.pdf. Acesso em: 03 jan. 2020.

ZUBERO, Imanol. La identidad cultural no existe. Madrid: Taurus, 2017.

\section{Juan Manuel López-Muñoz}

Doctor en Filología Francesa por la Universidad de Cádiz y profesor titular del Departamento de Filología Francesa e Inglesa en la Facultad de Filosofia y Letras de la Universidad de Cádiz.

\section{Maria da Glória Corrêa di Fanti}

Doctora en Lingüistica Aplicada y Estudios del Lenguaje en la Pontificia Universidade Católica de São Paulo (PUCSP) y profesora-investigadora en el Programa de Posgrado en Letras de la Facultad de Humanidades de la Pontificia Universidade Católica de Rio Grande do Sul (PUCRS). Becaria de Productividad de Investigación del CNPq.

\section{Bárbara Luzia Covatti Malcorra}

Doctoranda em Letras por la Pontificia Universidade Católica de Rio Grande do Sul (PUCRS). Becaria del CNPq. Máster en Letras por la misma institución. Licenciada em Letras (especialidad de Portugués e Inglés) por la Universidad de Vale do Rio dos Sinos (UNISINOS).

\section{Dirección:}

Juan Manuel López-Muñoz

Universidad de Cádiz (UCA)

Av. Dr. Gómez Ulla, 1

Cádiz 11003

Espanha

Maria da Glória Corrêa di Fanti

Pontifícia Universidade Católica do Rio Grande do Sul (PUCRS)

Av. Ipiranga, 6681, Prédio 8

Partenon 90619-900

Porto Alegre, RS, Brasil

Bárbara Luzia Covatti Malcorra

Pontificia Universidade Católica do Rio Grande do Sul (PUCRS)

Av. Ipiranga, 6681, Prédio 8

Partenon, 90619-900

Porto Alegre, RS, Brasil 